\title{
BMJ Open Preoperative clinical model to predict myocardial injury after non-cardiac surgery: a retrospective analysis from the MANAGE cohort in a Spanish hospital
}

Ana Belen Serrano (D) , ${ }^{1}$ Maria Gomez-Rojo, ${ }^{1}$ Eva Ureta, ${ }^{1}$ Monica Nuñez, ${ }^{1}$ Borja Fernández Félix, ${ }^{2}$ Elisa Velasco, ${ }^{3}$ Javier Burgos, ${ }^{4}$ Ekaterine Popova (D) , Gerard Urrutia, ${ }^{6}$ Victoria Gomez, ${ }^{4}$ Jose Manuel del Rey, ${ }^{7}$ Alfonso Sanjuanbenito, ${ }^{8}$ Javier Zamora, ${ }^{2,9,10}$ Juan Manuel Monteagudo, ${ }^{3}$ David Pestaña, ${ }^{1,11}$ Basilio de la Torre, ${ }^{12}$ Ángel Candela-Toha ${ }^{1}$

To cite: Serrano AB, GomezRojo M, Ureta $\mathrm{E}$, et al. Preoperative clinical model to predict myocardial injury after non-cardiac surgery: a retrospective analysis from the MANAGE cohort in a Spanish hospital. BMJ Open 2021;11:e045052. doi:10.1136/ bmjopen-2020-045052

- Prepublication history for this paper is available online. To view these files, please visit the journal online (http://dx.doi. org/10.1136/bmjopen-2020045052).

Received 20 September 2020 Accepted 12 July 2021

Check for updates

(c) Author(s) (or their employer(s)) 2021. Re-use permitted under CC BY-NC. No commercial re-use. See rights and permissions. Published by BMJ.

For numbered affiliations see end of article.

Correspondence to

Dr Ana Belen Serrano;

anab_serrano@yahoo.es

\section{ABSTRACT}

Objectives To determine preoperative factors associated to myocardial injury after non-cardiac surgery (MINS) and to develop a prediction model of MINS.

Design Retrospective analysis.

Setting Tertiary hospital in Spain.

Participants Patients aged $\geq 45$ years undergoing major non-cardiac surgery and with at least two measures of troponin levels within the first 3 days of the postoperative period. All patients were screened for the MANAGE trial. Primary and secondary outcome measures We used multivariable logistic regression analysis to study risk factors associated with MINS and created a score predicting the preoperative risk for MINS and a nomogram to facilitate bed-side use. We used Least Absolute Shrinkage and Selection Operator method to choose the factors included in the predictive model with MINS as dependent variable. The predictive ability of the model was evaluated. Discrimination was assessed with the area under the receiver operating characteristic curve (AUC) and calibration was visually assessed using calibration plots representing deciles of predicted probability of MINS against the observed rate in each risk group and the calibration-in-the-large (CITL) and the calibration slope. We created a nomogram to facilitate obtaining risk estimates for patients at pre-anaesthesia evaluation.

Results Our cohort included 3633 patients recruited from 9 September 2014 to 17 July 2017. The incidence of MINS was $9 \%$. Preoperative risk factors that increased the risk of MINS were age, American Status Anaesthesiology classification and vascular surgery. The predictive model showed good performance in terms of discrimination (AUC $=0.720 ; 95 \% \mathrm{Cl}: 0.69$ to 0.75 ) and calibration slope $=1.043(95 \% \mathrm{Cl}: 0.90$ to 1.18$)$ and $\mathrm{CITL}=0.00(95 \%$ Cl: -0.12 to 0.12$)$.

Conclusions Our predictive model based on routinely preoperative information is highly affordable and might be a useful tool to identify moderate-high risk patients before surgery. However, external validation is needed before implementation.
Strengths and limitations of this study

- The main strength of the present study is its large sample size.

- The simplicity and transposability of our predictive model allow its implementation worldwide.

- The main limitation is its unicentric setting, so our model needs external validation.

- The absence of preoperative troponin levels measurements may have favoured the inclusion of patients with chronically elevated troponin.

- Retrospective analysis and recollection of some data values in our study entail the usual limitations of observational studies with regard to potential confounders.

\section{INTRODUCTION}

Perioperative cardiovascular (CV) events are the leading cause of morbidity and mortality in patients undergoing major non-cardiac surgery. ${ }^{1}$ These events include cardiac death, acute myocardial infarction, cardiogenic pulmonary oedema, ventricular fibrillation, cardiac arrest and complete heart block. Preoperative risk estimation of CV events is based on validated models. Among them, the Revised Cardiac Risk Index ${ }^{2}$ (RCRI) has been widely used over the last 20 years probably due to its simplicity. The increasing availability of more specific and sensitive myocardial injury biomarkers, ${ }^{3}$ together with a deeper understanding of the pathophysiology of myocardial injury after major non-cardiac surgery (MINS), has paved the way to the recognition as a specific entity. ${ }^{4}$ The pathophysiology of MINS is multifactorial. Plaque rupture and mismatch between oxygen supply and 
demand have been considered as its main cause. ${ }^{35}$ Hyper/hypotension, hypoxia, sympathetic hyperactivity and inflammation are some of the aetiologies leading to thrombosis that can contribute to MINS. It has been proposed as a more suitable term for surgical patients ${ }^{6}$ since it is defined as a presumably ischaemic troponin elevation occurring within 30 days after surgery. ${ }^{7}$

It has been estimated that one out of every seven surgical patients older than 45 years with increased CV risk suffers from MINS. ${ }^{2}$ A meta-analysis published in 2011 showed that MINS was a strong independent mortality predictor within the first year after surgery. ${ }^{8}$ Moreover, a linear correlation between the elevation of troponin and mortality ${ }^{90}$ has been found. Therefore, MINS is now considered a complication of great significance ${ }^{11}$ leading to poor prognosis ${ }^{12}$ that may affect millions of patients after surgery worldwide. ${ }^{1}$ However, diagnosis is frequently missed out due to the effect of analgesic drugs commonly used as part of the immediate postoperative treatment, a reason why the vast majority of patients with MINS remain asymptomatic. This is why routine postoperative measurement of troponin has been proposed as a useful strategy to identify MINS among the surgical population. ${ }^{13} 14$ It would be highly beneficial to detect patients at moderatehigh risk of MINS and target preventive measures which ultimately could improve clinical outcomes after surgery. To date, there is no specific treatment for MINS either. However, recently some authors have also suggested strategies for its diagnosis and management. ${ }^{15}$ Many perioperative risk factors have been associated with postoperative MINS $^{67}$ such as older age, atrial fibrillation (AF), coronary artery disease (CAD), peripheral vascular disease and stroke. However, some other as $\mathrm{CV}$ risk factors (hypertension, diabetes mellitus, hypercholesterolemia, current history of smoking and obesity), the type of surgery (abdominal, urologic, orthopaedic, gynecologic, thoracic) or common $\mathrm{CV}$ medication taken by patients before surgery (acetyl salicylic, other antiplatelet drugs, statins, anticoagulant drugs), have not been properly assessed and might also be risk factors (or protectors) for MINS. In this study, we aimed to estimate the incidence of postoperative MINS in our setting and to develop a clinical prediction model for postoperative MINS.

\section{PARTICIPANTS AND METHODS}

\section{Study design and data collection}

A prospective cohort of 3633 patients aged $\geq 45$ years undergoing major non-cardiac surgery was retrospectively analysed for the development of MINS.

We gathered demographic (age and gender) and anthropometric (weight and height) data from medical records. We also collected clinical information regarding ASA classification ${ }^{16}$ and CV risk factors (hypertension, diabetes, obesity, hypercholesterolemia and smoking story). Hypertension, diabetes, hypercholesterolemia and current smoking were considered when they were documented in the clinical history. Obesity was defined

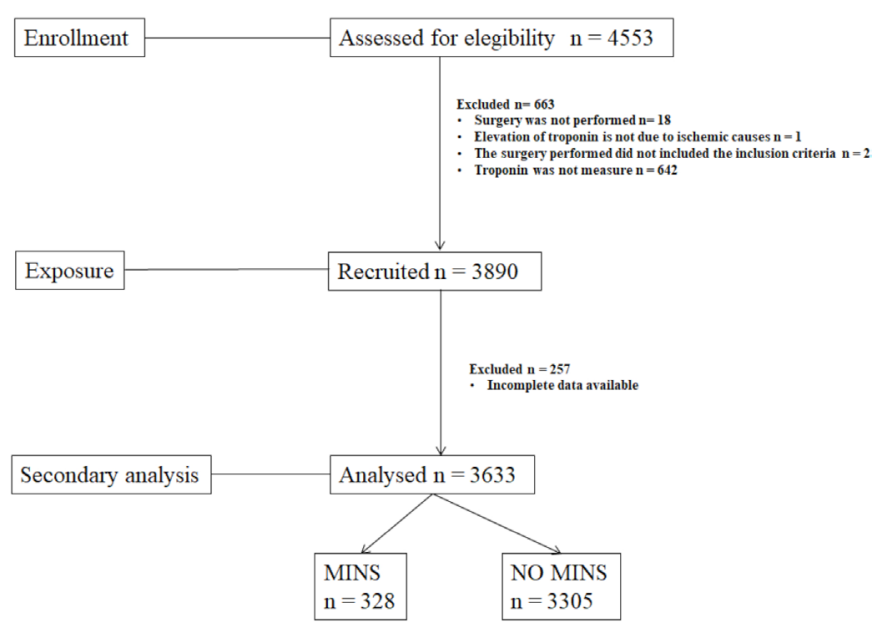

Figure 1 Flow chart of patients. MINS: myocardial injury after non-cardiac surgery.

for body mass index (BMI) $\geq 30$. We classified surgeries into abdominal, urological, traumatological-orthopaedic, gynaecological, vascular and thoracic surgeries. We also collected on preoperative medication taken at the moment of the anaesthesiologist visit.

The text adheres to the Strengthening the Reporting for OBservational in Epidemiology) ${ }^{17}$ statement.

\section{Study population}

The study population corresponds to patients screened in Ramón y Cajal University Hospital to participate in the MANAGE ${ }^{18}$ trial (ClinicalTrials.gov, number NCT01661101) from 9 September 2014 to 17 July 2017 (figure 1). The exposure in this study was undergoing major non-cardiac surgery. In the MANAGE ${ }^{18}$ trial, major non-cardiac surgery was defined as any surgery with an expected hospital length of stay for at least 2 days after surgery, and one of the following surgeries: abdominalaortic surgery, peripheral vascular surgery, laparotomy, major orthopaedic surgery of the hip or spine and thoracotomy. All participants underwent postoperative serum sampling and troponin concentrations were measured twice during the first 3 days after surgery.

Patients were eligible for MANAGE if they fulfilled all of the following criteria:

1. Undergone non-cardiac surgery.

2. $\geq 45$ Years of age.

3. Have suffered MINS based on fulfilling one of the following criteria: (A) elevated troponin or CK-MB (Creatine Kinase - Myocardial Band) measurement with one or more of the following features: (1) ischaemic signs or symptoms; (2) development of pathologic $\mathrm{Q}$ waves; (3) ECG changes indicative of ischaemia (ie, ST segment elevation, ST segment depression or inversion of $\mathrm{T}$ waves); (4) new LBBB (left bundle branch block) or (5) new or presumed new cardiac wall motion abnormality on echocardiography or new or presumed new fixed defect on radionuclide imaging. (B) elevated troponin measurement after surgery with no 
alternative explanation (eg, pulmonary embolism) to MINS.

4. Provide written informed consent to participate within 35 days of suffering their MINS.

In our study, we considered patients developed MINS only when an increased troponin occurred due to presumable ischaemic cause, or an isolated increased troponin occurred without an alternative explanation (ie, sepsis, pulmonary embolism or patients who underwent any cardiac resuscitation). Chronically elevated troponin measurement was not detected, because preoperative measurement of troponin not required by MANAGE study was not included.

\section{Perioperative cardiac troponin}

Regarding the troponin assay, conventional troponin I (cTnI) was measured from the beginning of the study using an Architect I200 Abbott Diagnostics analyser by mass immunoassay, being the cut-off point for MINS $0.1 \mathrm{ng} / \mathrm{mL}$. High sensitive troponin I (hsTnI) replaced cTnI measurement in June 2016, and this has been the routinely performed test from that moment on. When using hsTnI, the cut-off point was $\geq 30 \mathrm{pg} / \mathrm{mL}$. If both types of troponins were measured in the same blood sample, we only considered the value of the hsTn.

\section{Outcomes}

We analysed the incidence of MINS within the first 3 days after surgery. We classified the study population into MINS group when cTnI was $\geq 0.1 \mathrm{ng} / \mathrm{mL}$ due to an ischaemic cause and no-MINS when it was under than $0.1 \mathrm{ng} /$ $\mathrm{mL}$ or when troponin elevation did not occur.

\section{Statistical analysis}

First, we performed a descriptive analysis, stratified by MINS to characterise the study population. Continuous variables were expressed as mean and SD while categorical variables were summarised as absolute and relative frequencies. Incidence of MINS was computed as the number of MINS cases over the total number of patients included, along with the Clopper-Pearson 95\% CI. Comparisons between MINS and no-MINS groups were made using Student's t-test or $\chi^{2}$ test for continuous or categorical variables, respectively. We admitted those comparisons as statistically significant where $p$ value was below 0.05 .

\section{Development of the predictive model}

A logistic regression model was used to analyse the risk of MINS associated to clinical and demographic factors. We derived a multivariable predictive model including all the variables under studies as we thought they are all clinically relevant. Given that we did not collect actual values of the BMI of patients, we decided not to progress with the analysis of this factor as categorical. A non-linear relationship between age (continuous) and log odds of MINS was assessed using simple transformations and restricted cubic splines.
We used least absolute shrinkage and selection operator (LASSO) method for model fitting. LASSO is a regression analysis method that performs simultaneously a variable selection and a penalisation of coefficients. The objective is to improve model performance while, at the same time, reducing model over-optimism. Over-optimism is one of the major criticisms to statistical modelling and using regularisation techniques much as LASSO, we increased model external validity by reducing the size of the set-off covariates included in the final model. This is done iteratively applying a shrinkage factor to shrink coefficients to diminish their impact on model predictions, even to null values making a covariate to be excluded from the final model. We selected the penalty parameter (lambda) that minimised expected model deviance. Confidence intervals for model coefficients were obtained by bootstrap sampling. We evaluated the discriminatory ability of the final predictive model by computing the C-statistic summarised as the area under receiver operating characteristic curve with 95\% CI. Final model calibration was assessed by visually inspecting calibration plots representing deciles of predicted probability of MINS against the observed rate in each risk group. We also calculated the calibration slope adjusting a logistic regression model with the linear predictor as the only covariable. A slope equals one represents perfect calibration. The calibrationin-the-large was calculated as the intercept coefficient of the logistic regression with the linear predictor as offset, being zero as the ideal value. We also calculated the ratio of expected to observed number of events $(\mathrm{E} / \mathrm{O})$.

Finally, a simple, easy-to-use nomogram was developed to calculate the predicted probability of MINS at the time of preanaesthetic visit. Analyses were performed using Stata Software V.16.0 with nomolog user-defined command. ${ }^{19}$

\section{Patient involvement}

No patients were involved in proposing the research question or the outcome measures, nor were they involved in the design or implementation of the study. No patients were asked to participate on interpreting or writing up of results. Patients who developed MINS were advised to visit a cardiologist after hospital discharge. In case they already were chronically followed-up by one, we insisted on scheduling earlier appointment and to inform doctors about their new condition. There are no plans to disseminate the results of the research to study participants.

\section{RESULTS}

\section{Characteristics of the patient population}

A cohort of 3633 patients undergoing non-cardiac surgery screened for the MANAGE trial in our institution from September 2014 to July 2017 was retrospectively analysed for the development of MINS within the first 3 days after surgery (figure 1). Overall, 328 patients $(9.0 \%$ (95\% CI: $8.1 \%$ to $10.0 \%)$ ) developed this complication. Among our study population of 1712 patients, $47.1 \%$ were men. Mean age 
Table 1 Comparison of the studied characteristics between patients with and without MINS

\begin{tabular}{|c|c|c|c|c|}
\hline Variables & $\begin{array}{l}\text { Overall population } \\
(n=3633)\end{array}$ & $\begin{array}{l}\text { No MINS } \\
(n=3305)\end{array}$ & $\begin{array}{l}\text { MINS } \\
(n=328)\end{array}$ & $P$ value \\
\hline \multicolumn{5}{|l|}{ Demographics } \\
\hline Male & $1712(47.1)$ & $1578(47.7)$ & $136(40.96)$ & 0.017 \\
\hline ASA classification & & & & $<0.001$ \\
\hline III & 1217 (33.5) & 1079 (32.7) & $138(42.1)$ & \\
\hline IV & $294(8.1)$ & $201(6.1)$ & $93(28.3)$ & \\
\hline $\begin{array}{l}\text { Presence of at least one } \\
\text { cardiovascular risk factor }\end{array}$ & 2996 (82.5) & $2702(81.8)$ & 294 (89.6) & $<0.001$ \\
\hline Hypercholesterolemia & $1387(38.2)$ & $1238(37.5)$ & $149(45.4)$ & 0.005 \\
\hline $\mathrm{BMI}>30$ & $710(19.5)$ & $663(20.1)$ & $47(14.3)$ & 0.013 \\
\hline Types of surgeries & & & & $<0.001$ \\
\hline Abdominal & $822(22.6)$ & $756(22.9)$ & $66(20.1)$ & \\
\hline Vascular & $159(4.4)$ & $131(4.0)$ & $28(8.5)$ & \\
\hline Orthopaedic-traumat & $2.122(58.4)$ & $1.910(57.8)$ & $212(64.6)$ & \\
\hline Others & $530(14.6)$ & $508(15.4)$ & $22(6.7)$ & \\
\hline Urology & 482 & 462 & 20 & \\
\hline Statines & $1250(34.4)$ & 1116 (33.8) & $134(40.8)$ & 0.010 \\
\hline Other antiplatelet & $145(4.0)$ & $127(3.8)$ & $18(5.5)$ & 0.147 \\
\hline Anticoagulants & $629(17.3)$ & $530(16.1)$ & $99(30.2)$ & $<0.001$ \\
\hline
\end{tabular}

ASA classification, American Status Anaesthesiology classification; BMI, body mass index; MINS, myocardial injury after non-cardiac surgery.

was 72.9 years. The more frequent ASA classification was II followed by III ( $55 \%$ and $33 \%$, respectively). A high proportion $(82.5 \%)$ of patients presented at least one CV risk factor (table 1). Traumatological-orthopaedic and abdominal surgeries were the most commonly performed interventions, the majority of them being elective cases (99\%).

\section{Candidate predictors}

Univariate analysis identified nine potential risk factors: (1) age (OR 1.05; 95\% CI: 1.04 to 1.06), (2) female (OR 1.3; $95 \%$ CI: 1.1 to 1.7), (3) ASA classification (ref ASA I-II; $\mathrm{OR}_{\mathrm{ASA}}$ III $2.7 ; 95 \%$ CI: 2.0 to $3.5 ; \mathrm{OR}_{\text {ASA IV }} 9.5 ; 95 \%$ CI: 6.9 to 13.1$),(4)$ presence of at least one of the assessed CV risk factors (OR $1.9 ; 95 \%$ CI: 1.3 to 2.8 ), (5) vascular surgery (OR 2.3; CI 1.5 to $3.5)$, (6) acetyl salicylic acid (OR 1.6; 95\% CI 1.2 to 2.1), (7) statins (OR 1.3CI 1.1 to 1.7$)$, (8) other antiplatelet medication (OR 1.4; 95\% CI: 0.8 to 2.3) and (9) anticoagulant drugs taken before surgery (OR 2.2; 95\% CI 1.7 to 2.9). Oppositely, we found that obesity decreased the risk of postoperatory MINS (OR 0.6; 95\% CI: 0.5 to 0.9 ).

\section{Development of MINS prediction model using LASSO regression analysis}

Multivariate regression carried out using complete data (n=3633) (table 2), showed only three variables with a statistically significant association with MINS and were included in our final prediction model: age (OR 1.02), vascular surgery $(\mathrm{OR} 1.71)$ and ASA classification $\left(\mathrm{OR}_{\mathrm{ASA} \text { III }} 2.1\right.$ and $\mathrm{OR}_{\mathrm{ASA} \mathrm{IV}}$ $6.4)$. 


\begin{tabular}{lll}
$\begin{array}{l}\text { Table } 2 \\
\text { model }\end{array}$ & Multivariate analysis of predictors of MINS. Final \\
\hline Variables & OR & $95 \%$ bootstrap $\mathbf{~ I ~}$ \\
\hline Age (years) & 1.02 & 1.01 to 1.03 \\
ASA classification & & \\
I-II & Ref. & \\
III & 2.10 & 1.58 to 2.97 \\
IV & 6.42 & 4.34 to 9.90 \\
Vascular surgery & 1.71 & 1.00 to 3.15 \\
\hline
\end{tabular}

The equation of the model risk for MINS was as

follows: probability $(\mathrm{MINS})=\exp (\mathrm{Y}) /(1+\exp (\mathrm{Y}))$ where

$\mathrm{Y}=-3.647+0.020 \times($ age -45$)+0.744$ (ASA III)+1.859 (ASA IV)+0.536

(vascular surgery).

ASA classification, American Status Anaesthesiology classification;

MINS, myocardial injury after non-cardiac surgery.

The apparent C-statistic for the model was $0.72(95 \%$ CI 0.69 to 0.75 ). The calibration plot (figure 2) showed an overall good connection between the predicted and observed risks, and the calibration slope was $1.04(95 \%$ CI 0.90 to 1.18 ).

The model is presented as a graphical calculator (nomogram) in figure 3 . The equation of the model risk for MINS was as follows:

Probability $(\mathrm{MINS})=\exp (\mathrm{Y}) /(1+\exp (\mathrm{Y}))$

where $\mathrm{Y}=-3.517+0.020 \times($ age -45$)+0.744($ ASA III $)+1.859$

(ASA IV) +0.536 (vascular surgery).

\section{DISCUSSION}

\section{Statement of principal findings}

Our study was designed to draft a predictive model of preoperative risks factors for MINS in patients $\geq 45$ years

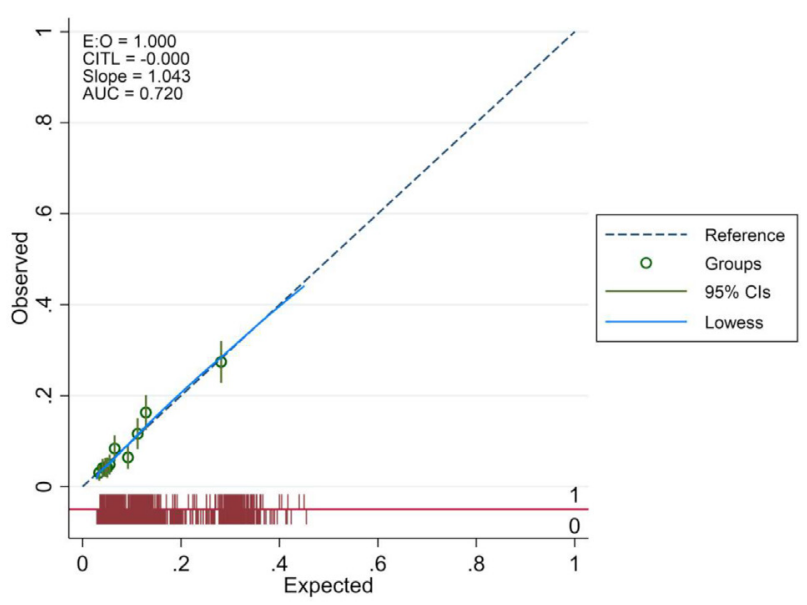

Figure 2 Calibration plot. Calibration plot representing expected and observed risk of MINS grouped by deciles of risk. Calibration slope $=1.04$ (95\% Cl: 0.90 to 1.18$)$, calibration in the large $=0.00(95 \% \mathrm{Cl}:-0.12$ to 0.12$)$ and C-statistic $(A \cup C)=0.72(95 \% \mathrm{Cl}: 0.69$ to 0.75$)$. AUC: area under the receiver operating characteristic curve; MINS: myocardial injury after non-cardiac surgery.
Nomogram
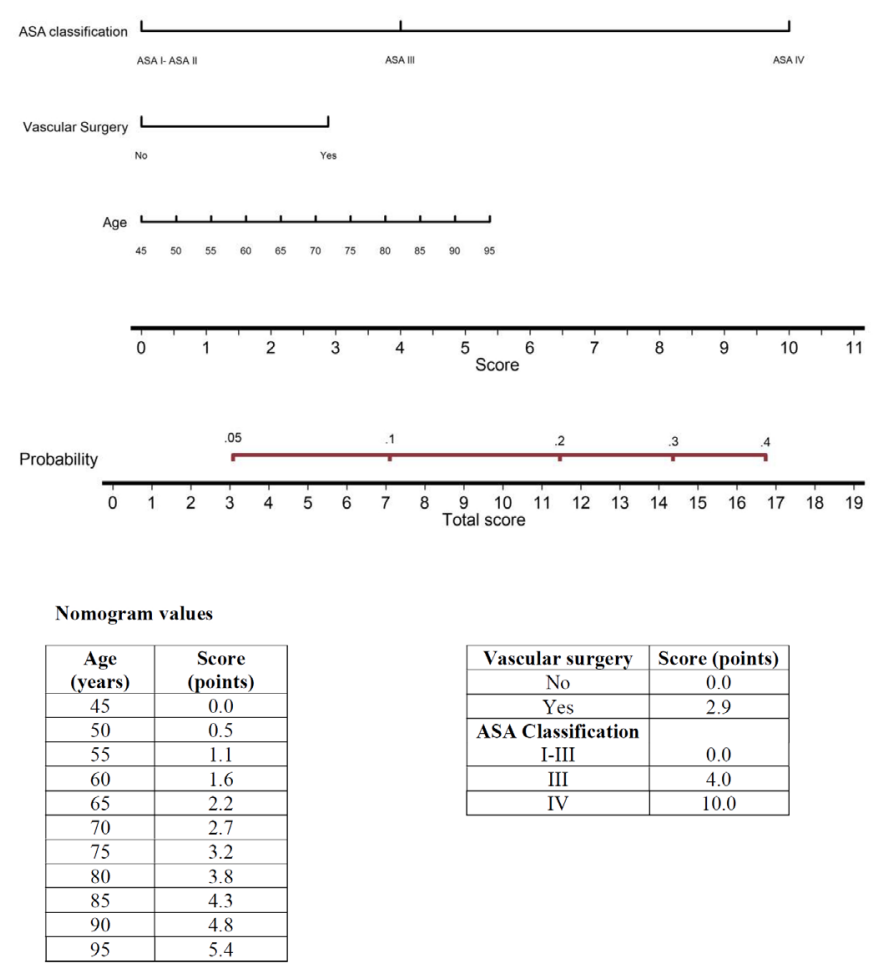

Figure 3 Nomogram of the predictive model for risk of MINS. To know the probability of developing MINS using this nomogram, three steps have to be taken. First, find out the value of each variable (ASA classification, whether vascular surgery is going to be performed or not and age) projecting a perpendicular line on the score line. Second, sum all these value scores and obtain a total score. Third, move to the total score line and project this value on the probability line. To obtain the percentage value, multiply it by 100 . For example, a patient ASA III, 50 years old who is going to vascular surgery has a probability of $11 \%$ to develop MINS as his total score is 7.4 points. ASA classification: American Status Anaesthesiology classification; MINS: myocardial injury after non-cardiac surgery.

undergoing a major non-cardiac surgery. We have identified three preoperative independent predictors of MINS: (1) age, (2) vascular surgery and (3) ASA scores III and IV. Based on these predictors we developed a risk score of MINS in order to stratify patients before surgery and to provide an estimated percentage risk for MINS.

The incidence of MINS in our cohort of 3633 patients undergoing major non-cardiac surgery was $9 \%$ a similar value to that published in the VISION study $(8 \%),{ }^{7}$ the largest cohort of surgical patients screened for myocardial injury. In other type of surgeries such as vascular procedures, this value becomes higher $(19.1 \%) .{ }^{20}$

\section{Comparison with other studies}

Perioperative cardiac risk estimation based on clinical risk indexes has been used for over 20 years, with the inception of the RCRI, long before the definition of MINS was established. In fact MINS is just one among many possible 
cardiac events, although notably, the most frequent. VISION investigators ${ }^{7}$ identified 12 independent preoperative predictors of MINS, doubling the number of risk factors included in the RCRI, including age (75 years or older), CV risk factors, known CV disease and surgical priority (urgent/emergent surgery). Known risk factors for developing MINS during the intraoperative period include excessive sympathetic stimulation, ${ }^{21}$ tachycardia and hypotension, ${ }^{22} 23$ surgery-related hypercoagulability, ${ }^{24}$ bleeding, ${ }^{25}$ hypothermia, ${ }^{26}$ hypoxia, pain ${ }^{27} 28$ and prolonged surgical time. House $e t a l^{29}$ developed a score to assess the impact of intraoperative haemodynamics in the development of myocardial injury. Moreover, other known postoperative independent risk factors of MINS are the development of acute kidney injury, the use of vasopressors, higher Acute Physiology and Chronic Health Evaluation II scores ${ }^{30}$ lower mean arterial pressure ${ }^{31}$ and hypoxemia. ${ }^{32}$ Durmuş $e t a l^{33}$ also found the neutrophil to lymphocyte ratio for a period of 3 days postoperatively can be used as an easy predictor of MINS. In an effort to improve the discrimination of RCRI to predict MINS, Douville $e t a l^{34}$ incorporated genetic information using a Polygenic Risk Score of CAD. Although associated with MINS, the addition of the genetic score did not improve discrimination. In order to develop our predictive model, we have focused on easily accessible and common clinical variables usually assessed in the preoperative period (age, gender, ASA classification, presence of hypertension, diabetes, hypercholesterolemia, current tobacco use, type of surgery and previous CV medication). The incorporation of intraoperative variables can certainly improve model prediction and discrimination. Although, the advantage of preoperative communication of risk to the patient is to some extent lost, the information obtained can be used to make decisions about measuring postoperative troponin. Measuring troponin routinely after surgery to diagnose MINS is still a matter of controversial debate. The ACC/AHA (American College of Cardiology/ American Heart Association) preoperative CV evaluation guidelines ${ }^{13}$ concluded that routine measurement of postoperative troponin levels in unselected patients without signs or symptoms of myocardial ischaemia or myocardial infarction is not useful for guiding perioperative management. On the contrary, Canadian Cardiovascular Society Guidelines on perioperative cardiac risk assessment and management for patients undergoing non-cardiac surgery ${ }^{14}$ "made a strong recommendation to obtain daily troponin measurements for 48-72 hours after surgery in patients with a baseline risk higher than $5 \%$ for CV deaths or nonfatal myocardial infarction at 30 days after surgery'. Measuring troponin seems appealing particularly in patients at high risk for MINS. The costs associated with a troponin $\mathrm{T}$ monitoring programme to detect MINS was found to be moderate. ${ }^{35}$ Also, Torborg et $a \hat{l}^{36}$ have published a pharmaco-economic analysis of routine postoperative troponin screening suggesting that such monitoring may be in fact cost-effective. We essentially agree with these findings although we insist on the importance of the need for tools that can provide early identification of the patients with risk of MINS, especially in settings where troponin measurement is not available.

There is no doubt on the theoretical relevance of these studies, but remains unclear whether all these new models have helped physicians in their daily clinical practice. Their complexity and sometimes the need for extra diagnostic tests count against them.

We present our predictive model and risk score of MINS as an easy and additional tool not only to identify patients at moderate to high risk of suffering this ischaemic complication, but with the intention to serve as a simple guide for clinical practice among surgical patients. Our risk score tool is easy to use and suitable for a vast majority of patients undergoing elective surgery worldwide. However, external validation is needed before implementation. Another feature of our model that has to be highlighted is the fact that it includes preoperative parameters in order physicians to have the chance of seeking counsel during both the intraoperative and postoperative periods. For example, making a bigger effort to avoid tachycardia, hypotension, anaemia and hypothermia during the intraoperative and postoperative periods or even targeting these goals in a narrower range in moderate to high risk patients can be considered an initial preventive step. Keeping anti-atherosclerosis medication should also be of potential benefit ${ }^{3738}$ in patients at moderate to high risk. Withholding aspirin ${ }^{15}$ and statins ${ }^{39}$ prior to surgery in high risk patients are other preventive approaches that are currently being assessed.

Our proposal is to avoid sampling troponin postoperatively in low risk patients as it is very unlikely that these will develop MINS but measuring it in patients with moderate to high risk. We consider patients are under moderate risk for MINS when they score a $10 \%$ estimated percentage risk according to our model; meaning an overall result of $>7$ in our score nomogram (figure 3). Therefore, this should be the cut-off point for both the screening of MINS by measuring troponin levels in the immediate postoperative period (if available) as well as for the implementation of potentially preventive measures throughout the perioperative period.

\section{Limitations}

This study was primarily limited by its unicentric setting what makes our results are not totally generalisable elsewhere, even though the sample size is large. In order to assure its universalisation, an external validation would be necessary. Futhermore, as we described in the Methods section, we only considered for MINS diagnosis troponin values from the immediate postoperative period, without knowing troponin value previous to surgery. So, we were unable to identify patients with chronically elevated troponin. This could be overcome by demonstrating proving uptrending or downtrending troponin values (especially with hsTn). However, we do not believe this is very likely as the MINS incidence found in our study was similar to some other important study published in the 
medical literature. ${ }^{7}$ Another limitation of our analysis was that retrospective analysis and recollection of some data values were performed despite The MANAGE study was conducted with a prospective design. This entailed the usual limitations of observational studies with regard to potential confounders. Including non-modifiable predictive factors in our model can be another limitation, as it precludes clinicians from using preoperative measures to reduce risk of MINS. Finally, because patient inclusion was dependent on investigator's availability, and some potential candidates may have been lost.

\section{Future research}

Consequently, due to the lack of information about both the prevention of MINS and its treatment, more studies with adequate design are needed in order to explore those issues and obtain further results.

\section{CONCLUSIONS AND CLINICAL IMPLICATIONS}

We have created a score for the prediction of the risk of postoperative MINS. Our proposal is to use it in all individuals undergoing non-cardiac major surgery in order to classify patients into low or moderate-high risk development of MINS. Patients at low risk should not be submitted to troponin sampling in the recent postoperative period. On the contrary, patients at moderatehigh risk of MINS should be monitored with troponin values whenever possible. Additionally, moderate-high risk patients should receive all the available preventive approaches during both intraoperative and postoperative period. In countries where troponin measurement is not fully available, this predictive model could also be used as a screening tool for MINS.

\section{Author affiliations}

${ }^{1}$ Department of Anesthesiology and Surgical Critical Care, Ramon y Cajal University Hospital. IRYCIS, Madrid, Spain

${ }^{2}$ Clinical Biostatistics Unit, Ramon y Cajal University Hospital. IRYCIS, Madrid, Spain ${ }^{3}$ Department of Cardiology, Ramon y Cajal University Hospital. IRYCIS, Madrid, Spain ${ }^{4}$ Department of Urology, Ramon y Cajal University Hospital. IRYCIS, Madrid, Spain ${ }^{5}$ Biomedical Research Institute, Iberoamerican Cochrane Center, (IIB Sant Pau), Barcelona, Catalunya, Spain

${ }^{6}$ CIBER Epidemiología y Salud Pública (CIBERESP), Biomedical Research Institute Sant Pau (IIB Sant Pau), Universitat Autònoma de Barcelona, Barcelona, Cataluña, Spain

${ }^{7}$ Department of Biochemistry, Ramon y Cajal University Hospital. IRYCIS, Madrid, Spain

${ }^{8}$ Department of General Surgery, Ramon y Cajal University Hospital. IRYCIS, Madrid, Spain

${ }^{9}$ CIBER Epidemiología y Salud Pública (CIBERESP), Madrid, Comunidad de Madrid, Spain

${ }^{10}$ Institute of metabolism and systems researchs, University of Birmingham,

Birmingham, UK

${ }^{11}$ Universidad Alcalá de Henares, Madrid, Spain

${ }^{12}$ Department of Traumatology, Ramon y Cajal University Hospital. IRYCIS, Madrid, Spain

Acknowledgements We gratefully acknowledge the contribution of Ana Castro as a data manager.

Contributors Study concept and design: ABS, AC-T and JZ. Acquisition of data: ABS, MN, EV, JB, AS, JMM, VG, GU, EP, JMdR, AS, BdIT, DP and EU. Analysis and interpretation of data: ABS, AC-T, JZ and BFF. Drafting of the manuscript: ABS, AC-T, $M G-R, J Z$ and $B F F$. The final manuscript was critically revised and approved by all authors.

Funding The authors have not declared a specific grant for this research from any funding agency in the public, commercial or not-for-profit sectors.

Competing interests None declared.

Patient and public involvement Patients and/or the public were not involved in the design, or conduct, or reporting, or dissemination plans of this research.

Patient consent for publication Not required.

Ethics approval The study was approved by the Ethical Committee of the Ramón y Cajal University Hospital, Madrid, Spain. Trial registration code in this institution LUXHOMBR001/2018.

Provenance and peer review Not commissioned; externally peer reviewed.

Data availability statement Data are available upon reasonable request. The data used in this study are part of a larger dataset. The data not used for this manuscript will be employed in future manuscripts.

Open access This is an open access article distributed in accordance with the Creative Commons Attribution Non Commercial (CC BY-NC 4.0) license, which permits others to distribute, remix, adapt, build upon this work non-commercially, and license their derivative works on different terms, provided the original work is properly cited, appropriate credit is given, any changes made indicated, and the use is non-commercial. See: http://creativecommons.org/licenses/by-nc/4.0/.

\section{ORCID iDs}

Ana Belen Serrano http://orcid.org/0000-0002-6370-5089

Ekaterine Popova http://orcid.org/0000-0002-8781-9873

\section{REFERENCES}

1 Devereaux PJ, Sessler DI. Cardiac complications in patients undergoing major noncardiac surgery. $N$ Engl J Med 2015;373:2258-69.

2 Lee TH, Marcantonio ER, Mangione CM, et al. Derivation and prospective validation of a simple index for prediction of cardiac risk of major noncardiac surgery. Circulation 1999;100:1043-9.

3 Iddagoda MT. The role of high-sensitive troponin measurement as a biomarker during the postoperative period for the detection of myocardial injury after non-cardiac surgery. $J$ Perioper Pract 2021;31:1-6.

4 Biccard BM. Detection and management of perioperative myocardial ischemia. Curr Opin Anaesthesiol 2014;27:336-43.

5 Smit M, Coetzee AR, Lochner A. The pathophysiology of myocardial ischemia and perioperative myocardial infarction. $J$ Cardiothorac Vasc Anesth 2020;34:2501-12.

6 Puelacher C, Lurati Buse G, Seeberger D, et al. Perioperative myocardial injury after noncardiac surgery: incidence, mortality, and characterization. Circulation 2018;137:1221-32.

7 Botto F, Alonso-Coello P, Chan MTV, et al. Myocardial injury after noncardiac surgery: a large, international, prospective cohort study establishing diagnostic criteria, characteristics, predictors, and 30day outcomes. Anesthesiology 2014;120:564-78.

8 Levy M, Heels-Ansdell D, Hiralal R, et al. Prognostic value of troponin and creatine kinase muscle and brain isoenzyme measurement after noncardiac surgery: a systematic review and meta-analysis. Anesthesiology 2011;114:796-806.

9 Devereaux PJ, Biccard BM, Writing Committee for the VISION Study Investigators, et al. Association of postoperative high-sensitivity troponin levels with myocardial injury and 30-day mortality among patients undergoing noncardiac surgery. JAMA 2017;317:1642-51.

10 Devereaux PJ, Chan MTV, Vascular Events In Noncardiac Surgery Patients Cohort Evaluation (VISION) Study Investigators, et al. Association between postoperative troponin levels and 30-day mortality among patients undergoing noncardiac surgery. JAMA 2012;307:2295-304.

11 Mauermann E, Puelacher C, Buse GL. Myocardial injury after non cardiac surgery: an underappreciated problem and current challenges. Curr Opin Anesthesiol 2016;29.

12 Beattie WS, Wijeysundera DN, Chan MTV, et al. Implication of major adverse postoperative events and myocardial injury on disability and survival: a planned subanalysis of the ENIGMA-II trial. Anesth Analg 2018;127:1118-26.

13 Fleisher LA, Fleischmann KE, Auerbach AD, et al. 2014 ACC/ AHA guideline on perioperative cardiovascular evaluation and 
management of patients undergoing noncardiac surgery: executive summary: a report of the American College of Cardiology/American Heart Association Task Force on Practice Guidelines. Circulation 2014;130:2215-45.

14 Duceppe E, Parlow J, MacDonald P, et al. Canadian cardiovascular Society guidelines on perioperative cardiac risk assessment and management for patients who undergo noncardiac surgery. Can $\mathrm{J}$ Cardiol 2017;33:17-32.

15 Devereaux PJ, Szczeklik W. Myocardial injury after non-cardiac surgery: diagnosis and management. Eur Heart J 2020;41:3083-91.

16 Abouleish AE, Leib ML, Cohen NH. ASA provides examples to each ASA physical status class. ASA Monitor 2015;79:38-9 http://monitor. pubs.asahq.org/article.aspx?articleid=2434536

17 von Elm E, Altman DG, Egger M, et al. Strengthening the reporting of observational studies in epidemiology (STROBE) statement: guidelines for reporting observational studies. BMJ 2007;335:806-8.

18 Devereaux PJ, Duceppe E, Guyatt G, et al. Dabigatran in patients with myocardial injury after non-cardiac surgery (MANAGE): an international, randomised, placebo-controlled trial. Lancet 2018;391:2325-34.

19 Zlotnik A, Abraira V. A general-purpose nomogram generator for predictive logistic regression models. Stata J 2015;15:537-46.

20 Biccard BM, Scott DJA, Chan MTV, et al. Myocardial injury after noncardiac surgery (MINS) in vascular surgical patients: a prospective observational cohort study. Ann Surg 2018;268:357-63.

21 Udelsman R, Norton JA, Jelenich SE, et al. Responses of the hypothalamic-pituitary-adrenal and renin-angiotensin axes and the sympathetic system during controlled surgical and anesthetic stress. $J$ Clin Endocrinol Metab 1987;64:986-94.

22 Abbott TEF, Pearse RM, Archbold RA, et al. A prospective international multicentre cohort study of intraoperative heart rate and systolic blood pressure and myocardial injury after noncardiac surgery: results of the VISION study. Anesth Analg 2018;126:1936-45.

23 van Waes JAR, van Klei WA, Wijeysundera DN, et al. Association between intraoperative hypotension and myocardial injury after vascular surgery. Anesthesiology 2016;124:35-44.

24 Rosenfeld BA, Beattie C, Christopherson R. The effects of different anesthetic regimens on fibrinolysis and the development of postoperative arterial thrombosis. Perioperative ischemia randomized anesthesia trial Study Group. Anesthesiology 1993;79:255-61.

25 Kamel H, Johnston SC, Kirkham JC, et al. Association between major perioperative hemorrhage and stroke or $\mathrm{Q}$-wave myocardial infarction. Circulation 2012;126:207-12.

26 Frank SM, Beattie C, Christopherson R, et al. Unintentional hypothermia is associated with postoperative myocardial ischemia. Anesthesiology 1993;78:468-76.
27 Mangano DT, Siliciano D, Hollenberg M, et al. Postoperative myocardial ischemia. therapeutic trials using intensive analgesia following surgery. the study of perioperative ischemia (SPI) Research Group. Anesthesiology 1992;76:342-53.

28 Beattie WS, Badner NH, Choi P. Epidural analgesia reduces postoperative myocardial ischemia: therapeutic trials using intensive analgesia following surgery. Anesthesiology 1992;76:342-53.

29 House LM, Marolen KN, St Jacques PJ, et al. Surgical Apgar score is associated with myocardial injury after noncardiac surgery. $J$ Clin Anesth 2016;34:395-402.

30 Smilowitz NR, Redel-Traub G, Hausvater A, et al. Myocardial injury after noncardiac surgery: a systematic review and meta-analysis. Cardiol Rev 2019;27:267-73.

31 van Lier F, Wesdorp FHIM, Liem VGB, et al. Association between postoperative mean arterial blood pressure and myocardial injury after noncardiac surgery. Br J Anaesth 2018;120:77-83.

32 Sun Z, Sessler DI, Dalton JE, et al. Postoperative hypoxemia is common and persistent: a prospective blinded observational study. Anesth Analg 2015;121:709-15.

33 Durmuș G, Belen E, Can MM. Increased neutrophil to lymphocyte ratio predicts myocardial injury in patients undergoing non-cardiac surgery. Heart Lung 2018;47:243-7.

34 Douville NJ, Surakka I, Leis A, et al. Use of a polygenic risk score improves prediction of myocardial injury after non-cardiac surgery. Circ Genom Precis Med 2020;13:e002817.

35 Buse GL, Manns B, Lamy A, et al. Troponin T monitoring to detect myocardial injury after noncardiac surgery: a cost-consequence analysis. Can J Surg 2018;61:185-94.

36 Torborg A, Ryan L, Kantor G, et al. The pharmacoeconomics of routine postoperative troponin surveillance to prevent and treat myocardial infarction after non-cardiac surgery. S Afr Med J 2014;104:619-23.

37 Chen JF, Smilowitz NR, Kim JT, et al. Medical therapy for atherosclerotic cardiovascular disease in patients with myocardial injury after non-cardiac surgery. Int J Cardiol 2019;279:1-5.

38 van Waes JAR, Grobben RB, Nathoe HM, et al. One year mortality, causes of death, and cardiac interventions in patients with postoperative myocardial injury. Anesth Analg 2016;123:29-37.

39 Berwanger O, Le Manach Y, Suzumura EA, et al. Association between pre-operative statin use and major cardiovascular complications among patients undergoing non-cardiac surgery: the VISION study. Eur Heart J 2016;37:177-85. 